\title{
El (neo)liberalismo en el documento de Justicia y Paz sobre la crisis económica y financiera
}

\author{
Ildefonso Camacho S.I., Facultad de Teología, Granada
}

Aunque no es un documento polémico, llama la atención en él las alusiones que se hacen al liberalismo o al neoliberalismo. Como eso no es frecuente en los textos de la doctrina social de la Iglesia, nos ha parecido de interés someter a consideración estas referencias y situarlas en el contexto más amplio de dicha doctrina social.

\section{Cinco referencias explícitas}

En cinco ocasiones se habla del liberalismo. Comenzaremos pasando revista a cada una de ellas, aunque no seguiremos el orden en que aparecen en el texto.

La primera tiene que ver con el origen de la crisis reciente, concretamente con la quiebra de algunos bancos norteamericanos, cuando todavía se pensaba que era mejor no acudir en rescate de instituciones que habían llegado a una situación tan delicada. Se prefería que el mercado resolviese el problema:

Una orientación de tipo liberal -reticente respecto a las intervenciones públicas en los mercados- ha propiciado la quiebra de un importante instituto internacional, imaginando de este modo, delimitar la crisis y sus efectos ${ }^{27}$.

La cita más importante es la que identifica al liberalismo económico explícitamente como uno de los dos factores que han impulsado al mundo hacia esta situación que se ha convertido incluso en una amenaza para la paz:

Ante todo, un liberalismo económico sin reglas y sin supervisión. Se trata de una ideo-
logía, de una forma de apriorismo económico, que pretende tomar de la teoría las leyes
del funcionamiento del mercado y las denominadas leyes del desarrollo capitalista,
exagerando algunos de sus aspectos. Una ideología económica que establezca a priori
las leyes del funcionamiento del mercado y del desarrollo económico, sin confrontarse
con la realidad, corre el peligro de convertirse en un instrumento subordinado a los

${ }^{27}$ CONSEJO PONTIFICIO JUSTICIA Y PAZ, Para una reforma del sistema financiero y monetario internacional en la perspectiva de una autoridad pública de ámbito mundial (24 de octubre de 2011), n. 1. 
intereses de los países que ya gozan, de hecho, de una posición de mayores ventajas económicas y financieras ${ }^{28}$.

Se acusa al liberalismo económico de ser un "apriorismo económico", porque se basa en la teoría económica sin confrontarse con la realidad: mientras que la teoría parece indicar que la libertad, entendida aquí como ausencia de reglas y de supervisión, es el mejor garante del progreso, la experiencia se encarga de mostrar que no siempre es así. Pero esta ceguera puede ocultar además una mala intención: la de legitimar los mecanismos que han permitido que se impongan los intereses de los países más poderosos, porque evidentemente la apuesta por una libertad con las restricciones mínimas favorece a los que tienen más poder.

La tercera cita tiene un carácter más indirecto: se menciona al liberalismo junto al utilitarismo, que es el segundo factor al que se responsabiliza de impulsar la crisis actual:

\begin{abstract}
A la base de las disparidades y de las distorsiones del desarrollo capitalista se encuentra en gran parte, además de la ideología del liberalismo económico, la ideología utilitarista es decir la impostación teórico-práctica según la cual "lo que es útil para el individuo conduce al bien de la comunidad". Es necesario notar que una "máxima" semejante, contiene un fondo de verdad, pero no se puede ignorar que no siempre lo que es útil individualmente, aunque sea legítimo, favorece el bien común. En más de una ocasión es necesario un espíritu de solidaridad que trascienda la utilidad personal por el bien de la comunidad ${ }^{29}$.
\end{abstract}

Tomamos en consideración esta mención porque la relación del utilitarismo con el liberalismo es fácil de establecer. El mismo texto lo insinúa al recordar el enfoque individualista que subyace al utilitarismo. Y apunta a uno de los problemas mayores que este individualismo liberal plantea: la imposibilidad de articular los intereses de los individuos con el bien común de la sociedad. Esta es una cuestión no ya estrictamente económica, sino abiertamente filosófica.

Una cuarta cita se refiere a la ideología tecnocrática, que ahora se pone en relación con el neoliberalismo. Consiste en eliminar la dimensión ética de los problemas, concretamente del desarrollo, reduciéndolo todo a pura técnica:

Para interpretar con lucidez la actual nueva cuestión social, es necesario evitar el error, hijo también de la ideología neoliberal, de considerar que los problemas por afrontar

\footnotetext{
$28 \mathrm{lbid}$.

$29 \mathrm{lbid}$.
} 
son de orden exclusivamente técnico. En cuanto tales, escaparían a la necesidad de un discernimiento y de una valoración de tipo ético ${ }^{30}$.

La tecnocracia se mueve en el campo de la razón instrumental, que se preocupa por el "cómo", por los medios, pero se desentiende del fin, del "para qué". Dicho de otra forma, se trata de poner la economía y las finanzas bajo la primacía de la ética y de la política, que viene a ser el mensaje de fondo de todo el documento y que se aplica sobre todo al hablar de la propuesta de establecer una autoridad política mundial ${ }^{31}$.

La última cita que encontramos remite a un texto de la encíclica Populorum progressio, en el que Pablo VI denuncia explícitamente los abusos del liberalismo económico:

Los peligros de una situación de desarrollo económico, concebido en términos de liberalismo, han sido denunciados lúcida y proféticamente por Pablo VI -a causa de las nefastas consecuencias sobre los equilibrios mundiales y la paz- ya en 1967, después del Concilio Vaticano II, con la Encíclica Populorum progressio. El Pontífice indicó, como condiciones imprescindibles para la promoción de un auténtico desarrollo, la defensa de la vida y la promoción del progreso cultural y moral de las personas. Sobre tales fundamentos, Pablo VI afirmaba que el desarrollo plenario y planetario "es el nuevo nombre de la paz"1"32.

Aunque de Populorum progressio se citan los números 76 y siguientes, que hablan del desarrollo como nombre de la paz y de la necesidad de una autoridad mundial, Pablo VI critica al liberalismo de una forma muy expresa a propósito del mercado libre aplicado al comercio internacional, del que tanto se beneficiaban los países industrializados frente a los exportadores de materias primas. Véase el pasaje:

Es decir que la regla del libre cambio no puede seguir rigiendo ella sola las relaciones internacionales. Sus ventajas son ciertamente evidentes cuando las partes no se encuentran en condiciones demasiado desiguales de potencia económica: es un estímulo del progreso y recompensa del esfuerzo. Por eso los países industrialmente desarrollados ven en ella una ley de justicia. Pero ya no es lo mismo cuando las condiciones son demasiado desiguales de país a país: los precios que se forman "libremente" en el mercado pueden llevar consigo resultados no equitativos. Es, por consiguiente, el principio fundamental del liberalismo, como regla de los intercambios comerciales, el que está aquí en litigio ${ }^{33}$.

30 Ibid., n. 2.

${ }^{31}$ Ibid., n. 4.

32 Ibid., n. 2.

${ }^{33}$ PABLO VI (1967) Populorum progressio, n. 58. 
Hemos citado este pasaje de Pablo VI completo porque expresa bien, ya en 1967, una de las razones por las que las conclusiones de la teoría económica sobre la eficacia del mercado no son válidas cuando se aplican a mercados reales donde las condiciones de partida de los agentes que intervienen son muy desiguales. Esta misma crítica hacía ya León XIII en 1891, pero entonces para referirse al mercado de trabajo, donde la desigual situación de patronos y obreros condenaba a estos últimos a condiciones laborales y sociales cada vez más miserables ${ }^{34}$.

A la luz de los cinco pasajes que hemos analizado, se puede concluir que el liberalismo es objeto de atención por parte del Consejo Pontificio Justicia y Paz. Lo entiende en primer término como liberalismo económico, pero sin olvidar el trasfondo filosófico individualista. A ello se une la mentalidad tecnocrática, que no es ajena en el fondo a este individualismo y que impedir entender la responsabilidad ética de los agentes económicos. La cita de Pablo VI permite también aceptar que este documento de ahora se siente en coherencia con la tradición anterior de la doctrina social, aunque en esta es más bien raro que se mencione explícitamente el liberalismo.

\section{Para iluminar el tema desde la doctrina social de la Iglesia}

Pero la no mención del liberalismo no quiere decir que este no esté implícito en muchos documentos sociales de la Iglesia. Mencionaremos tres referencias importantes: de León XIII, de Pablo Vl y de Juan Pablo II; prescindiremos, en cambio, de Benedicto XVI porque su doctrina está más presente entre nosotros.

Suele colocarse como comienzo de la doctrina social de la Iglesia la encíclica Rerum novarum que publicara León XIII en 1891. Pero este no es su primer documento oficial. Hasta cinco encíclicas sobre cuestiones políticas publicó León XIII antes de Rerum novarum y otras dos posteriormente ${ }^{35}$. De todas ellas nos interesa

\footnotetext{
${ }^{34}$ Puede verse en texto en: LEÓN XIII (1891) Rerum novarum, n. 32. Pablo VI lo recuerda justamente a continuación del pasaje que hemos citado (n. 59).

${ }^{35}$ Son anteriores: Diuturnum illud (1881), sobre el origen del poder; Humanum genus (1884), sobre la francmasonería; Immortale Dei (1885), sobre la constitución cristiana del Estado; Libertas praestantissimum (1888), sobre la libertad humana y el liberalismo; Sapientiae christianae (1890), sobre los deberes del ciudadano cristiano. Y todavía otras dos después: Au milieu des sollicitudes (1892), sobre las formas de gobierno, dirigida al pueblo francés para clarificar la postura concreta de los católicos ante la república recientemente instaurada; Graves de communi (1901), sobre el auténtico sentido que
} 
para nuestro propósito la encíclica Libertas praestantissimum, que es un análisis y toma de postura sobre el liberalismo: se estudia su esencia, sus formas y las llamadas "libertades modernas". Y se hace contraponiendo dos concepciones de la libertad para concluir mostrando las consecuencias prácticas de esa comparación. El liberalismo se critica por su concepción de la libertad: una libertad que no se somete a ninguna instancia superior, ni reconoce verdad alguna, divina ni humana, a la que someterse. León XIII reconoce que esta es la expresión más radical del liberalismo y le contrapone la concepción cristiana de la libertad, caracterizada por la subordinación del ser humano a una verdad que está por encima de él. Naturalmente esa verdad es sobre todo de orden moral.

Esta crítica de la libertad, que se refiere ante todo a los aspectos filosóficos, será retomada por Juan Pablo II en Centesimus annus para explicar las reservas de la Iglesia frente al sistema político del capitalismo: la democracia pluralista. La democracia se cree con derecho a definir la verdad (de nuevo aquí se está pensando en la verdad moral) desde el simple juego de las mayorías parlamentarias, sin asumir que hay principios éticos de orden natural a la que todo régimen político debe someterse:

Hoy se tiende a afirmar que el agnosticismo y el relativismo escéptico son la filosofía y la actitud fundamental correspondientes a las formas políticas democráticas, y que cuantos están convencidos de conocer la verdad y se adhieren a ella con firmeza no son fiables desde el punto de vista democrático, al no aceptar que la verdad sea determinada por la mayoría o que sea variable según los diversos equilibrios políticos. A este propósito hay que observar que si no existe una verdad última, la cual guía y orienta la acción política, entonces las ideas y las convicciones humanas pueden ser instrumentalizadas fácilmente para fines de poder. Una democracia sin valores se convierte con facilitad en un totalitarismo visible o encubierto, como demuestra la historia ${ }^{36}$.

Resumiendo la posición de Juan Pablo II: el sistema político de capitalismo (la democracia partidista) no es rechazable en sí; lo es el concepto de libertad que lo alimenta. Pero las referencias a la libertad en esta encíclica no terminan ahí. También el sistema económico del capitalismo (la economía de mercado) es criticado: y de nuevo no por el mercado en sí, sino por la concepción de libertad que subyace, una libertad que pone en primer término el aspecto económico (la libertad de actuación económica). El pasaje más significativo merece ser leído a pesar de su extensión:

hay que dar entre los católicos a la democracia cristiana.

36 JuAN PABlo II (1991) Centesimus annus, n. 46. El papa cita como apoyo en este pasaje la encíclica Libertas. Esta encíclica de León XIII es citada en varias ocasiones más a lo largo de nuestro texto. 
Volviendo ahora a la pregunta inicial ¿̇se puede decir quizá que, después del fracaso del comunismo, el sistema vencedor sea el capitalismo, y que hacia él estén dirigidos los esfuerzos de los países que tratan de reconstruir su economía y su sociedad? (...) La respuesta obviamente es compleja. Si por "capitalismo" se entiende un sistema económico que reconoce el papel fundamental y positivo de la empresa, del mercado, de la propiedad privada y de la consiguiente responsabilidad para con los medios de producción, de la libre creatividad humana en el sector de la economía, la respuesta ciertamente es positiva, aunque quizá sería más apropiado hablar de "economía de empresa", "economía de mercado" o simplemente de "economía libre". Pero si por "capitalismo" se entiende un sistema en el cual la libertad, en el ámbito económico, no está encuadrada en un sólido contexto jurídico que la ponga al servicio de la libertad humana integral y la considere como una particular dimensión de la misma, cuyo centro es ético y religioso, entonces la respuesta es absolutamente negativa ${ }^{37}$.

Ahora sí se está hablando del aspecto económico de la libertad. Juan Pablo II no utiliza el término "liberalismo", pero no parece arriesgado decir que tiene muy presente a la ideología liberal, con su exaltación de la libertad individual.

¿Es esta postura tan descarnada la que inspira a todo liberalismo en nuestro tiempo? Probablemente no. Y esto nos lleva a la tercera referencia que queríamos hacer, que es anterior en el tiempo a Juan Pablo II: la carta Octogesima adveniens de Pablo VI (1971). Buscando unos criterios éticos para el compromiso de los cristianos con la democracia echa mano de la distinción entre ideologías y movimientos históricos, que ya usara Juan XXIII para iluminar las posibles vías de colaboración de los creyentes con los que no lo son. Si las ideologías son más inflexibles en sus principios y como inmutables en el tiempo, se revisten de una mayor flexibilidad cuando los movimientos históricos inspirados por ellas buscan aplicarlas a los problemas concretos de cada época ${ }^{38}$.

Cuando Pablo Vl aborda concretamente las ideologías de nuestro tiempo (marxismo y liberalismo), se refiere a la ideología liberal en estos términos:

Tampoco apoya el cristiano la ideología liberal, que cree exaltar la libertad individual sustrayéndola a toda limitación, estimulándola con la búsqueda exclusiva del interés y del poder, y considerando las solidaridades sociales como consecuencias más o menos automáticas de las iniciativas individuales y no ya como fin y motivo primario del valor de la organización social ${ }^{39}$.

37 Ibid., n. 42. Puede verse también el n. 39.

${ }^{38}$ Cf. PABLO VI (1971) Octogesima adveniens, n. 30, que reproduce lo que decía JUAN XXIII (1963) Pacem in terris, n. 159.

${ }^{39}$ PABLO VI (1971) Octogesima adveniens, n. 26. 
Los motivos del rechazo son dos: poner como valores supremos la libertad ilimitada y la búsqueda exclusiva del poder. Pero además se cuestiona también el individualismo, expresado ahora como resistencia a que las solidaridades sociales sean asumidas como objetivo directo de la organización de la sociedad: ¿̇no es esta una constante, incluso hoy, del liberalismo, tan reacio a articular esos objetivos sociales como tareas del Estado?

Pero esta crítica no obsta para admitir que el cristiano pueda implicarse en movimientos históricos (por ejemplo, los partidos) liberales, con tal que lo haga desde un atento discernimiento para sopesar el grado de compromiso en función de valores y desvalores. Como valores del liberalismo: la eficiencia económica, la iniciativa personal, la voluntad de defender al individuo contra el dominio cada vez más invasor de las organizaciones y las tendencias totalitarias de los poderes políticos. Como desvalores, la excesiva autonomía que se concede al individuo en su actividad, en sus motivaciones y en el ejercicio mismo de su libertad ${ }^{40}$.

Pero Pablo VI se ha referido más explícitamente al liberalismo económico en su encíclica Populorum progressio (1967). Aquí sí hay un rechazo muy explícito del capitalismo liberal:

Pero, por desgracia, sobre estas nuevas condiciones de la sociedad ha sido construido un sistema que considera el provecho como motor esencial del progreso económico, la concurrencia como ley suprema de la economía, la propiedad privada de los medios de producción como un derecho absoluto, sin límites ni obligaciones sociales correspondientes. Este liberalismo sin freno que conduce a la dictadura, justamente fue denunciado por Pío $\mathrm{XI}$ como generador del imperialismo internacional del dinero ${ }^{41}$.

Para entender este texto hay que fijarse en los adjetivos, que subrayan cómo el liberalismo tiende a dar al lucro, a la competencia y a la propiedad privada una consideración sin límites: por eso se habla de motor esencial, de ley suprema, de derecho absoluto.

Esta crítica se aplica luego al comercio internacional, donde se producían las mayores injusticias contra los países menos desarrollados, de nuevo como consecuencia de una forma de entender la libertad que privilegia a los que más poder tienen (en este caso, económico y comercial). El texto, que quedó citado más arriba apuntaba

\footnotetext{
${ }^{40} \mathrm{Ibid}$, n. 35. Una argumentación paralela se aplica a la ideología marxista (ibid.) y a los movimientos históricos socialistas y marxistas (ibid., nn. 31-34).

${ }^{41}$ PABLO VI (1967) Populorum progressio, n. 26.
} 
ya, hace casi 50 años, lo que hoy es el gran problema de la globalización: la liberalización de los mercados a nivel mundial cuando los agentes que intervienen en él tienen distinto poder y margen de libertad y no existe ninguna instancia que vele por los legítimos intereses de los más débiles.

\section{Conclusión}

El reciente documento de Justicia y Paz está en franca continuidad con una tradición que la doctrina social de la Iglesia ha ido desarrollando desde el siglo XIX. Prescindiendo de los aspectos más polémicos, que corresponden a momentos de mayor radicalismo por ambas partes, la lglesia ha marcado en su doctrina oficial un claro distanciamiento respecto al liberalismo, que tiene dos motivos fundamentales: la concepción de la libertad y, como trasfondo, el individualismo. Estas discrepancias afectan esencialmente a la filosofía liberal, pero no se circunscriben a ella: alcanza también a las concreciones de esta en las instituciones políticas y económicas.

Durante mucho tiempo estas discrepancias se han ceñido al modelo vigente en las sociedades particulares. La doctrina de la Iglesia ha contribuido a hacer evolucionar el capitalismo liberal y el Estado liberal de Derecho hacia el capitalismo mixto y el Estado social. Para ello ha insistido en dos puntos. El primero se refiere a la libertad: porque, cuando no todos los ciudadanos disponen de ella en igualdad de condiciones, el resultado es que aumentan esas desigualdades de partida; por eso son precisas instancias correctoras de esos mecanismos. En segundo lugar, ello supone que una concepción de la sociedad basada en el individualismo no es suficiente: porque la sociedad puede y debe establecer objetivos sociales que orienten a los individuos en función de los intereses de todos, y no solo para paliar los periuicios de los más débiles, sino para establecer cauces de colaboración entre todos.

El documento que comentamos eleva esa doctrina del nivel más particular en que se ha gestado al nivel planetario. Porque la crisis y el contexto globalizador en que se ha producido ponen de manifiesto que la sociedad actual ya no se entiende si no es como sociedad mundial. Y ahí los problemas son idénticos y las soluciones tienen que ir por un camino parecido al mencionado: la libertad tiene que ser sometida a cauces, también en lo económico (y financiero), y es preciso establecer objetivos planetarios que eviten lucha salvaje por la supervivencia y garanticen la convivencia de todos los pueblos. 
Pero las dimensiones de esa sociedad mundial son una dificultad añadida, tanto para suscitar la solidaridad y la colaboración como para constituir una instancia con capacidad para formular esos objetivos y actuar eficientemente en su consecución. Por eso el documento puede resumirse en dos propuestas concretas: la necesidad de crear una autoridad política mundial -una idea que ya lanzó Juan XXIII y ha recogido Benedicto XVI en su encíclica social ${ }^{42}$ y la sugerencia de que sea la regulación de los mercados financieros una de la primeras tareas -si no la primera- que esta autoridad acometa.

${ }^{42}$ Cf. Juan XXIII (1963) Encíclica Pacem in terris, nn. 131-141 y Benedicto XVI (2009) Encíclica Caritas in veritate, $\mathrm{n} .67$ 